\title{
Neutrino Majorana Mass and Baryon Number of the Universe below the Electroweak Symmetry breaking Scale
}

\author{
H.V. Klapdor-Kleingrothaus ${ }^{\dagger}$, St. Kolb ${ }^{\dagger}$ and U. Sarkar ${ }^{\dagger, \ddagger}$ \\ † Max-Planck-Institut für Kernphysik, P.O. 1039 80, D-69029 Heidelberg, \\ Germany \\ $\ddagger$ Physical Research Laboratory, Ahmedabad, 380009 India
}

\begin{abstract}
If the neutrino is Majorana type and the electroweak phase transition is second or weak first order, neutrino-induced interactions together with sphaleron transitions have the potential to erase a previously generated baryon asymmetry of the universe. Taking correctly into account the evolution of the vacuum expectation of the Higgs field the effective light neutrino masses are constrained to be lighter than $\mathcal{O}(10 \mathrm{MeV})$, while the effective heavy masses are constrained to be heavier than $\mathcal{O}\left(10^{7} \mathrm{GeV}\right)$.
\end{abstract}

Recent experiments seem to indicate that the neutrino is massive [1]. From a model-building point of view the most natural structure of the neutrino mass matrix contains both Lepton-number- $(L-)$ conserving Dirac- and $L$-violating Majoranatype entries (see e.g. [2]). In principle observable consequences are $L$-violating processes such as neutrinoless double beta $(0 \nu \beta \beta)$ decay and $L$-violating leptongauge boson scattering (inverse $0 \nu \beta \beta$ decay).

On the other hand, in the early universe $L$ violation together with sphaleronmediated transitions has the potential to create the Baryon number $(B)$ of the universe (BAU) (Leptogenesis) or erase an existing BAU. The latter may be the case both above or below the electroweak symmetry breaking scale. This consideration would limit the amount of $L$ violation and hence give a bound on the Majorana mass of the neutrinos. In the following we will reconsider the limits on neutrino masses and point out that the evolution of the vacuum expectation value (vev) of the Higgs responsible for electroweak symmetry breaking weakens the existing bound by as large as three orders of magnitude.

In an extension of the standard model, the Majorana mass of the neutrinos can come from an effective dimension-5 operator [3]

$$
\frac{\alpha_{i j}}{M}\left(L_{i}^{T} C^{-1} \tau_{2} \vec{\tau} L_{j}\right)\left(H^{T} \tau_{2} \vec{\tau} H\right)
$$


where $i, j=1,2,3$ are generation indices, $M$ is the $L$-violating mass-scale, $\alpha$ is an effective coupling and $L(H)$ are $S U(2)_{L}$ lepton (Higgs) doublets. After the electroweak symmetry breaking, when the higgs doublet scalar acquires a vev, the neutrinos get a Majorana mass of the order of

$$
m_{\nu} \sim \frac{\alpha_{i j}\langle v(T)\rangle^{2}}{M}
$$

where $\langle v(T)\rangle$ is the vev of $H$. The condition that the associated $L$ violation should not wash out the primordial BAU then gives an upper bound on the Majorana mass of the neutrinos of the order of a few keV [4, 5]. In most of these approaches the analysis was simplified by assuming the vev to be constant and the rate of the $L$-violating interactions was considered to be less than the expansion rate of the universe. However, above the critical temperature $T_{C}$ of the electroweak phase transition (EWPT) the vev of $H$ is zero. Below $T_{C}$ the $v e v$ starts growing. On the other hand soon the sphalerons freeze out and they cannot wash out the BAU any longer. Thus, during the period when the sphalerons wash out the BAU, the vev may still be quite small. This weakens the upper bound on the Majorana mass of the neutrinos. In the following this argument will be discussed in more detail.

Consider the see-saw mechanism of neutrino masses [6]. The $L$-violating mass scale will have its origin from integrating out heavy $S U(2)_{L}$ singlet neutrinos. The neutrino mass-matrix has the general structure

$$
\mathcal{M}^{\nu}=\left(\begin{array}{cc}
0 & \left(m^{D}\right)^{T} \\
m^{D} & M
\end{array}\right)
$$

where $m^{D} \sim\langle v(T)\rangle$ is the Dirac mass matrix coupling $S U(2)_{L}$ doublet neutrinos $\nu$ to $S U(2)_{L}$ singlet neutrinos $N$, whereas $M$ is a Majorana mass matrix for the $N$ 's. Below the EWS the Higgs field aquires a (temperature dependent) vacuum expectation value $\langle v(T)\rangle$ and so that $\nu$ and $N$ mix with resulting masses

$$
m_{i}=\sum_{j} U_{i j} \mathcal{M}_{i j}, U U^{\dagger}=1
$$

If the scale of $M$ is much larger than that of $m^{D}$ the diagonal mass-matrix consists of two blocks of light and heavy masses (see-saw mechanism)

$$
m_{\text {light }} \approx-\left(m^{D}\right)^{T} M^{-1} m^{D}, m_{\text {heavy }} \approx M .
$$

The off-diagonal blocks of the mixing matrix $U$ are approximately $\left(m^{D}\right)^{\dagger}\left(M^{-1}\right)^{\dagger}$ and $-M^{-1} m^{D}$.

In the triplet higgs model [7] one introduces a triplet higgs scalar $\xi$ with mass $M$. The couplings of the triplet higgs breaks lepton number explicitly at the scale 
$M$, but the vev of the triplet higgs gets a see-saw contribution of amount

$$
\langle\xi\rangle \sim \frac{\langle H\rangle^{2}}{M} .
$$

The direct coupling of the triplet higgs with the two neutrinos then give a Majorana mass to the neutrinos.

In the early universe neutrinos give rise to $L$-violating processes such as $(i, j$ are generation indices)

$$
e_{i}^{ \pm} e_{j}^{ \pm} \leftrightarrow W^{ \pm} W^{ \pm}
$$

The masses $m_{i j}^{M}$ give rise to $L$-violating processes such as neutrinoless double beta decay $(0 \nu \beta \beta)$ (for an overview see e.g. [8]). For a linear collider this process has been studied in [9].

In the early universe $m_{i j}^{M}$ induced processes have the potential to erase the obeserved asymmetry in the baryon- $(B)$ and antibaryon-number of the universe (BAU) (see e.g. [10]). This is due to the fact that as long as sphaleron transitions are in thermal equilibrium 12 $B$ and $L$ are both proportional to $(B-L)$ 11. Hence, if $L$ is erased by an $L$-violating process and sphalerons are still operative, $B$ is erased as well. If the electroweak phase transition (EWPT) with an associated critical temperature $T_{C}$ is strong first order sphalerons are never in thermal equilibrium and $L$-violating processes do not affect $B$ below $T_{C}$. On the other hand, if the EWPT is second or weak first order there is a period

$$
T_{\text {out }}<T<T_{C}
$$

( $T_{\text {out }}$ denotes the sphaleron freezing-out temperature) during which $L$-violating processes have the potential to erase $L$ and consequently $B$. Hence, if the EWPT is second or weak first order the requirement that a preexisting BAU should not be washed out poses a limit on the amount of $L$ violation. In the case of a Majorana neutrino in previous works [4] an estimated limit $m_{i j}^{M} \lesssim 20 k e V$ has been obtained. In this note it will be argued that this bound is in fact three orders of magnitude less stringent if the temperature dependence of the vev is correctly taken into account as has been done recently for the case of $L$-violating sneutrinos in [13].

The temperature dependence of the $v e v$ for a second or weak first order phase transition is given by

$$
\langle v(T)\rangle \approx\langle v(T=0)\rangle\left(1-T^{2} / T_{C}^{2}\right)^{1 / 2},\langle v(T=0)\rangle=246 G e V
$$

and the the sphaleron rate in the broken phase is [14]

$$
\Gamma_{S p h}(T) \approx 2.8 \cdot 10^{5} T^{4} \kappa\left(\frac{\alpha_{W}}{4 \pi}\right)^{4}\left(\frac{2 m_{W}(T)}{\alpha_{W} T}\right)^{7} \exp \left(-\frac{E_{s p}(T)}{T}\right)
$$




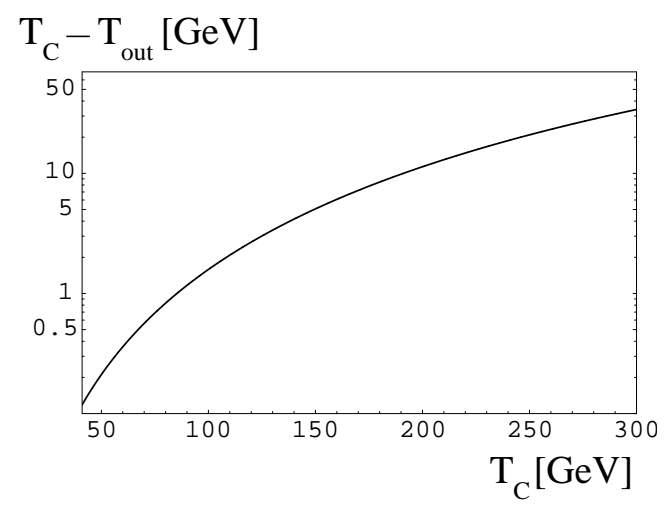

Figure 1: The difference of the critical temperature $T_{C}$ and the sphaleron freezing out temperature $T_{\text {out }}$ determined by $\Gamma_{\text {sph }}\left(T_{\text {out }}\right)=H\left(T_{\text {out }}\right)$ in dependence of $T_{C}$.

where

$$
m_{W}(T)=\frac{1}{2} g_{2}\langle v(T)\rangle
$$

the free energy of the sphaleron configuration is given by

$$
E_{S p h}(T)=\frac{2 m_{W}(T)}{\alpha_{W}} B\left(\frac{m_{H}}{m_{W}}\right),
$$

$B(0)=1.52, B(\infty)=2.72$ and $\kappa=\exp (-3.6)$ [15]. As usual $T_{\text {out }}$ is determined by the condition

$$
\Gamma_{\text {sph }}\left(T_{\text {out }}\right)=H\left(T_{\text {out }}\right)=1.7 \sqrt{g_{*}} \frac{T_{\text {out }}^{2}}{M_{P l}}
$$

where $M_{P l} \approx 10^{19} \mathrm{GeV}$ is the Planck scale and $g_{*} \approx 100$ in the Standard Model. Lattice simulations suggest that for a Higgs mass of around $m_{H} \approx 70 \mathrm{GeV} T_{C} \approx$ $150 \mathrm{GeV}$ and higher for larger values of $m_{H}$ [16]. For our phenomenological purposes $T_{C}$ will be varied between $50 \mathrm{GeV}$ and $250 \mathrm{GeV}$. The temperature range eq. (7) is plotted in figure田. It is smaller than $1 \mathrm{GeV}$ for $T_{C} \lesssim 100 \mathrm{GeV}$ but of order $\mathcal{O}(10 \mathrm{GeV})$ for $T_{C} \gtrsim 200 \mathrm{GeV}$.

Relevant processes for depleting a pre-existing $L$ number during the epoch (77) are $L$-violating $2 \leftrightarrow 2$ scatterings $W^{ \pm} W^{ \pm} \leftrightarrow e_{i}^{ \pm} e_{j}^{ \pm}, W^{ \pm} e_{i}^{\mp} \leftrightarrow W^{\mp} e_{j}^{ \pm}$and gauge boson decays. The depletion of an initial $L$ number $L_{i}$ is described by (see for example [10])

$$
\left.L(z)=L_{i} \exp \left[-\int_{z_{c}}^{z_{\text {out }}} d z^{\prime} z^{\prime}\left[g_{*} \frac{n_{\tilde{\nu}}}{s} \Gamma_{D}\left(z^{\prime}\right)+n_{\gamma}\langle\sigma|v|\rangle\right)\right] / H\left(T=m_{\tilde{\nu}}\right)\right]
$$



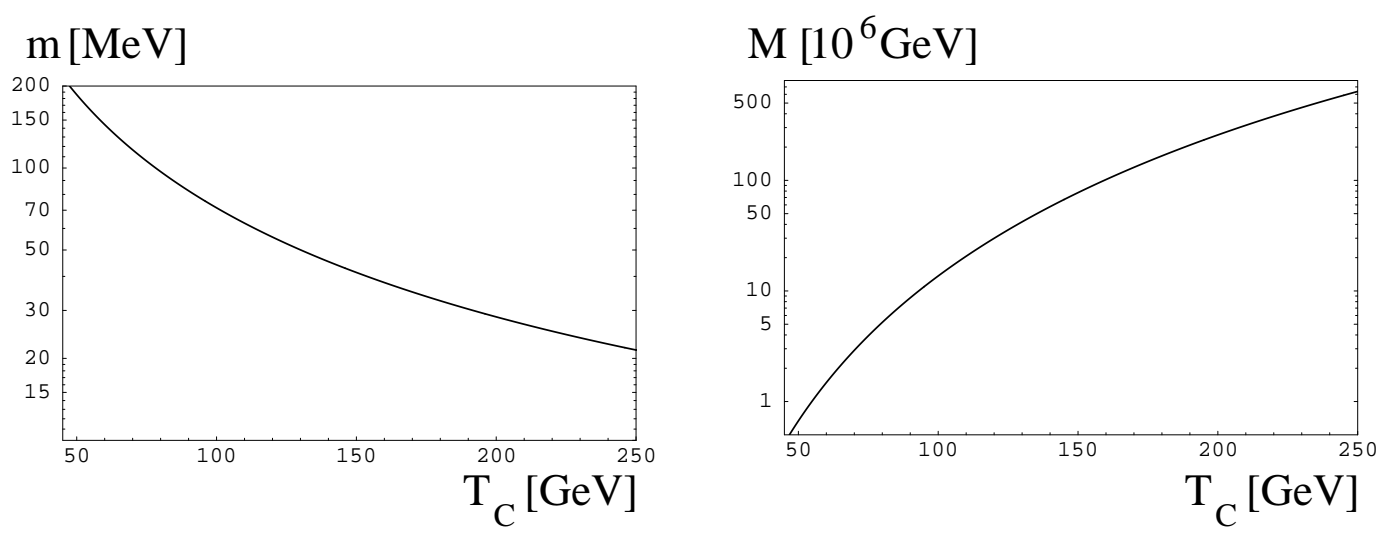

Figure 2: The bounds on the superposition of light neutrino states $\left\langle m_{i j}\right\rangle$ (left, region above the curve excluded) and of heavy neutrino states $\left\langle M_{i j}\right\rangle$ (right, region below the curve excluded)

Working with the Boltzmann equation will then give us the amount of residual asymmetry after the spheleron transitions have frozen out. Unlike earlier works, where the interaction rate has been compared with the expansion rate of the universe, we consider the condition for erasure of the primordial BAU is that the asymmetry depletes by a factor of at least 10. In most of the cases when we get the bound, the depletion is more than two orders of magnitude.

For the see-saw mechanism case the thermally averaged contribution of the light neutrino states with masses $m_{k} \ll T_{C}$ may be approximated by

$$
\langle\sigma|v|\rangle_{i j} \approx \frac{\alpha_{W}^{2}\left\langle m_{i j}\right\rangle^{2}}{T^{4}},\left\langle m_{i j}\right\rangle=\sum_{k} U_{i k} U_{j k} m_{k}
$$

and the contribution of the heavy states with masses $M_{n} \gg T_{C}$ is

$$
\langle\sigma|v|\rangle \approx \frac{\alpha_{W}^{2}}{\left\langle M_{m n}\right\rangle^{2}}, \frac{1}{\left\langle M_{i j}\right\rangle}=\frac{\sum_{n} U_{i n} U_{j n}}{M_{n}}
$$

where $\alpha_{W}$ is the weak coupling constant. Compared to the zero-temperature case both cross-sections are suppressed by a factor

$$
\frac{\langle v(T)\rangle^{2}}{\langle v(T=0)\rangle^{2}}=\left(1-\frac{T^{2}}{T_{C}^{2}}\right)^{2}
$$

since for the light states $m_{k} \sim\langle v(T)\rangle^{2}$ and for the heavy states $U_{i n} U_{j n} \sim\langle v(T)\rangle^{2}$, see above. The bounds on the quantities $\left\langle m_{k}\right\rangle$ and $\left\langle M_{n}\right\rangle$ are displayed in figure 
2. For plausible values of the critical temperature $T_{C} \gtrsim 150 \mathrm{GeV}$ the bound on the light states is of order $\left\langle m_{i j}\right\rangle \lesssim \mathcal{O}(10 \mathrm{MeV})$ while the bound on the heavy states is of order $\left\langle M_{i j}\right\rangle \gtrsim 10^{7} \mathrm{GeV}$.

For the triplet higgs mechanism, the bound on the mass of the neutrino comes out to be the same as the bound on the light neutrino state of the see-saw mechanism. In this case also the suppression is given by equation (16), since the neutrino mass is again proportional to $m_{\nu} \propto\langle v(T)\rangle^{2}$. Thus even in this case the bound comes out to be around $10 \mathrm{MeV}$. Given the generality of the dimension-5 operators for the Majorana neutrino mass, one may conclude that in all models of neutrino masses this bound is valid. Previous estimates of the light neutrino masses have been given in [4] for the case eq. (1) and it has been argued that for every entry of the corresponding mass matrix the bound from the BAU is of order $m_{i j} \lesssim 10 k e V$, that is three orders of magnitude more stringent than if the evolution of the vev is taken into account.

In summary, we included the effect of evolution of the higgs vev and solved the Boltzmann equation in estimating the bound on the neutrino masses coming from the erasure of the baryon asymmetry of the universe. This makes the bounds three orders of magnitude weaker than the one obtained from earlier naive estimates.

\section{Acknowledgement}

U.S. wants to thank Max-Planck-Institut für Kernphysik for hospitality.

\section{References}

[1] Y. Fukuda et al., the Super-Kamiokande Collaboration, Phys. Lett. B 467 (1999) 185.

[2] R.N. Mohapatra and P.B. Pal, "Massive Neutrinos in Physics and Astrophysics" (World Scientific, Singapore, 1991).

[3] S. Weinberg, Phys. Rev. Lett. 43 (1979) 1566; E. Akhmedov, Z. Berezhiani and G. Senjanovič, Phys. Rev. Lett. 69 (1992) 3013; E. Ma, Phys. Rev. Lett. 43 (1998) 1171.

[4] M. Fukugita and T. Yanagida, Phys. Rev. D 42 (1990) 1285; S.M. Barr and A.E. Nelson, Phys. Lett. B 246 (1991) 141; W. Fischler, G. Giudice, R. Leigh and S. Paban, Phys. Lett. B 258 (1991) 45; W. Buchmüller T. and Yanagida, Phys. Lett. B 302 (1993) 240; U. Sarkar, Phys. Lett. B 390 (1997) 97.

[5] B. Campbell, S. Davidson, J. E. Ellis and K. Olive, Phys. Lett. B 256 (1991) 457; E. Ma, M. Raidal and U. Sarkar Phys. Lett. B 460 (1999) 359 H. Dreiner and G.G. Ross, Nucl. Phys. B 410 (1993) 188; J.M. Cline, K. Kainulainen and 
K.A. Olive Phys. Rev. D 49 (1994) 6394; A. Ilakovac and A. Pilaftsis, Nucl. Phys. B 437 (1995) 491.

[6] M. Gell-Mann, P. Ramond and R. Slansky, in Supergravity, Proceedings of the Workshop, Stony Brook, New York, 1979, ed. by P. van Nieuwenhuizen and D. Freedman (North-Holland, Amsterdam); T. Yanagida, in Proc of the Workshop on Unified Theories and Baryon Number in the Universe, Tsukuba, Japan, 1979, edited by A. Sawada and A. Sugamoto (KEK Report No. 79-18, Tsukuba); R.N. Mohapatra and G. Senjanović, Phys. Rev. Lett. 44 (1980) 912.

[7] E. Ma and U. Sarkar, Phys. Rev. Lett. 80 (1998) 5716; G. Lazarides and Q. Shafi, Phys. Rev. D 58 (1998) 071702.

[8] H.V. Klapdor-Kleingrothaus in Proc. Int. Conf. on Lepton- and Baryon-Number Non-Conservation, Trento, Italy, April 20-25, 1998, IOP, Bristol (1999); H.V. Klapdor-Kleingrothaus, Int. J. Mod. Phys. A 13 (1998) 3953.

[9] G. Belanger, F. Boudjema, D. London and H. Nadeau, Phys. Rev. D 53 (1996) 6292; C. Greub and P. Minkowski, Int. J. Mod. Phys. A 13 (1998) 2363.

[10] E.W. Kolb and M.S. Turner, "The Early Universe" (Addison-Wesley, Redwood City, CA, 1990).

[11] S. Yu. Khlebnikov and M.E. Shaposhnikov, Nucl. Phys. B 308 (1988) 885; J.A. Harvey and M.S. Turner, Phys. Rev. D 42 (1990) 3344.

[12] V.A. Kuzmin, V.A. Rubakov and M. Shaposhnikov, Phys. Lett. B 155 (1985) 36.

[13] H.V. Klapdor-Kleingrothaus, St. Kolb and V.A. Kuzmin, Phys. Rev. D, in press and hep-ph/9909546.

[14] V.A. Rubakov and M.E. Shaposhnikov, Usp. Fiz. Nauk 166 (1996) 493, Phys. Usp. 39 (1996) 461; A. Riotto, hep-ph/9807454.

[15] G.D. Moore, Phys. Rev. D 59 (1999) 014503.

[16] K. Kajantie, M. Laine, K. Rummukainen and M. Shaposhnikov, Nucl. Phys. B 466 (1996) 189. 